\title{
Correspondence
}

\section{Cleaning and disinfection of equipment for gastrointestinal flexible endoscopy: interim recommendations of a Working Party of the British Society of Gastroenterology}

SIR, - The report by the Working Party (Gut 1988; 29: 1134-51) has been drawn to our attention and in particular, the section regarding management of HIV infected patients. The authors incorrectly state that 'there is no information on the activity of common disinfectants against the intermediate forms and cysts of cryptosporidium'. The relevance of 'intermediate forms' - that is, endogenous or tissue stages, in transmission is uncertain. The inherent resistance of the transmissible form of the parasite, the oocyst, to disinfection has been known for some considerable time. ${ }^{1-4}$ Attempts have been made to extend the earlier studies using a combination of an in vitro test system, based on excystation inhibition, and in vivo verification of loss of infectivity (unpublished data). As a result, guidelines have been drawn up. (In preparation.)

Table Efficacy of physical and chemical agents for the disinfection of oocysts of Cryptosporidium

(1) Chemical Methods (30 mins at ambient temperature*)

(i) Fully effective -10 volume hydrogen peroxide

(ii) Partially effective - Exspor ${ }^{\mathbb{B}}$ (Alcide) NWD $\dagger$

$1 \%$ sodium hypochlorite ${ }^{\dagger}$

$1 \%$ ammonia

$1 \%$ sodium hydroxide

(iii) Ineffective - $2 \%$ activated gluteraldehyde $1 \%$ formalin $1 \%$ Phenol, $2 \%$ Hycolin ${ }^{\circledR}, 4 \%$ lysol $90 \%$ ethanol, iso-propanol, $\mathrm{N}$-propanol $10 \%$ povidone iodine

$0.5 \%$ potassium permanganate Presept ${ }^{\circledR}$ NWD

Sporicidin ${ }^{\circledR}$ NWD

$5 \%$ Dettol $^{\circledR}$

(2) Physical methods

(i) Heat inactivation - oocysts have a low thermal death point and can be killed by five to 10 minutes at $50-55^{\circ} \mathrm{C}$. Oocysts are also killed by freezing.

(ii) Oocysts are inactivated by drying.

*Some compounds may show increased effect with increased temperature.

†Inhibited by presence of protein.

NWD: Normal recommended working dilution.

${ }^{\circledR}$ : Registered trade name.

Evidence has been adduced for symptomless oocyst excretion in normal subjects, asymptomatic carriage detected by intubation, and apparent reactivation of infection which may have resulted from such carriage. Given the resistance of the oocysts, the frequency with which the infection is known to occur in the immunologically normal human population and the potential seriousness of the infection in the immunocompromised, effective disinfection of endoscopes is essential.5 Adequate cleaning may considerably reduce the level of risk. Given the above findings, however, the recommendation for disinfection by means of aldehyde preparations may not deal adequately with oocysts of Cryptosporidium and further studies are required. Such studies are in hand.

D P CASEMORE

Public Health Laboratory,

Glan Clwyd Hospital,

Bodelwyddan,

Rhyl,

Clwyd LL18 5UJ,

and

D A BLEWETT AND S E WRIGHT

Animal Diseases Research Association,

Moredun Research Institute,

408 Gilmerton Road,

Edinburgh EH17 7JH

\section{References}

1 Angus KW, Sherwood D, Hutchison G, Campbell I. Evaluation of the effect of two aldehyde-based disinfectants on the infectivity of faecal cryptosporidia for mice. Res Vet Med 1982; 33: 379-81.

2 Campbell I, Tzipori S, Hutchison G. Angus KW. Effect of disinfectants on survival of cryptosporidium oocysts. Vet Rec 1982; 111: 414-5.

3 Angus KW. Cryptosporidiosis in man, domestic animals and birds: a review. J $R$ Soc Med 1983; 76: 62-70.

4 Tzipori S. Cryptosporidiosis in animals and humans. Microbiol Rev 1983; 47: 84-96.

5 Casemore DP. Human cryptosporidiosis. In: Reeves D. Geddes A, eds. Recent advances in infection $\mathrm{No}_{0} 3$. Edinburgh: Churchill Livingstone, 1989: 209-236.

\section{Reply}

SIR, - Casemore et al provide important information on the activity of disinfectants and cryptosporidial oocysts. It is difficult to see how our interim recommendations on endoscope disinfection should be modified.

When considering disinfectants for use in endoscopy a variety of factors have to be considered. These include the antimicrobial activity against a spectrum of organisms likely to be present as contaminants, staff toxicity, and danger of hypersensitivity, and damage to endoscopic equipment.

Hydrogen peroxide has been used to flush through the internal channels of endoscopes to clear protein 
debris. It may, however, damage external surfaces and in particular the rubbers and plastics of the insertion tube so instrument manufacturers do not recommend immersion. Casemore and Blewett provide evidence that it is fully effective if cryptosporidial oocysts are exposed for 30 minutes. Flushing and shorter times may not be sufficient.

There are problems with the four other disinfectants listed and which are said to be partially effective. Exspor in preliminary tests against polio virus and herpes simplex virus has shown rather poor activity compared with glutaraldehyde (Ayliffe G. personal communication). One per cent ammonia is not suitable for bacterial disinfection, sodium hypochlorite damages endoscopes and sodium hydroxide may do also.

Although in immunocompetent individuals cryptosporidium causes a transient diarrhoea, the infection may be life threatening in the immunosuppressed. While there have been no reports of endoscopic transmission we would agree that a new disinfectant, active against cryptosporidium, is needed for use before endoscopy in immunosuppressed patients. At present we recommend thorough mechanical cleaning followed by immersion for one hour in $2 \%$ activated glutaraldehyde before and after endoscopy on immunocompromised patients. This is to ensure that atypical mycobacteria are not transmitted to immunosuppressed patients and that $\mathrm{M}$ tuberculosis is not transmitted from a symptomatic patient with HIV infection to an immunocompetent patient.

Casemore and Blewett provide evidence that 30 minutes in $2 \%$ glutaraldehyde is not effective for cryptosporidium. We stress in our report that thorough mechanical cleaning with detergent is the most important part of the disinfection procedure. It would seem that we have to rely on this until there is an alternative disinfectant. We hope that the work underway by Casemore et al will contribute to final recommendations.

I V D WELLER, A T R AXON, AND D J JEFFRIES Gastroenterology Unit, General Infirmary, Leeds

\section{Epidemiological study of asymptomatic inflammatory bowel disease}

SIR, - Dr Mayberry and colleagues (Gut 1989; 30: 481-3) used prevalence rates in the above article in a rather misleading way. For instance, comparisons in their Table 4 do not state which age groups the prevalence rates refer to in the various studies which they have compared. The use of age specific rates would have overcome this difficulty.

\section{Dept of Community Medicine, Lothian Health Board, Edinburgh}

F NAMDARAN

SIR, - The paper by Mayberry et al is interesting and also a timely reminder of what significant pathology may exist undetected in our communities.

It would be interesting to know how the Nottingham group decided to manage these asymptomatic patients and how well their patients complied with any treatments suggested.

\section{Northern General Hospital, Sheffield}

J A R SMITH

\section{Reply}

SIR, - We would like to thank both correspondents for their comments. The use of 'age specific rates' for the previously published data reported in Table 4 would have been inappropriate as they refer to the prevalence of inflammatory bowel disease in whole populations. Unfortunately, as discussed in the article published, age specific prevalence rates for populations aged 50-74 are unavailable.

Both patients with Crohn's disease underwent surgical resection. This would not be our routine practise in asymptomatic patients but there was concern that the abnormalities detected on radiological examination could have been tumours. All patients with ulcerative colitis were treated with sulphasalazine and are now regularly followed in an inflammatory bowel disease clinic.

J F MAYBERRY AND K C BALLANTYNE

Leicester General Hospital,

Leicester and

Queens Medical Centre,

Nottingham

Comparison of forceful dilatation and oesophagomyotomy in achalasia

SIR, - The paper in the March issue (Gut 1989; 30: 299$304)$ by Csendes and colleagues is of great interest. Pneumatic dilatation for the management of achalasia in their hands did not perform as well as surgical management though as they admit their results using the pneumatic method are not as good as those of others. We have now followed up a much larger group of patients than they refer to with the results remaining as good. ' Their disappointing results may be related to the much lower inflation pressures which they use.

My impression is that many physicians/gastroenterologists are managing achalasia non-surgically. I consider this a welcome development for the very 